\title{
A DISPERSIVE CONTINUUM MODEL OF JOINTED MEDIA
}

\author{
BY
}

S. SHKOLLER (University of California San Diego, La Jolla, CA)

A. MAEWAL (Trans Science Corporation, La Jolla, CA)

AND

G. A. HEGEMIER (University of California San Diego, La Jolla, CA)

\begin{abstract}
The problem of formulating higher-order continuum models for a jointed medium is considered for propagation of waves whose wavelengths are much larger than the cell width. A multiscale asymptotic approach is used to derive exact solutions for the microstructure in this large wavelength limit. A mixed variational principle is then invoked to obtain the homogenized model. This model, which incorporates dispersive wave phenomena, yields results which agree well with the exact solution for the dispersion of harmonic waves propagating through the medium.
\end{abstract}

1. Introduction. In this paper we consider wave propagation through a jointed medium whose domain consists of a matrix material subdivided by periodically spaced joints. The solution of the differential equation that describes the behavior of this material must, evidently, satisfy appropriate continuity conditions at the matrix-joint interfaces. Consequently, the equation is equivalent, in the distributional sense, to a differential equation whose coefficients are periodic functions of the spatial independent variable. Since it is often quite difficult to obtain a numerical or analytic solution of such problems, especially if the number of joints is large, it is of interest to homogenize this differential equation.

We wish to obtain an equivalent differential equation with constant coefficients whose solution is in some sense close to that of the original problem. The approach, of course, will depend on the nature of the applied excitations, and we limit our scope to forcing functions whose Fourier components have wavelengths much larger than the length of periodicity. To obtain the homogenized equation valid for small values of $\varepsilon$, the ratio of the length of periodicity to wavelength, we use a multiscale asymptotic technique.

The method is based upon the introduction of two length scales: a microcoordinate - the fast spatial variable corresponding to the microstructure-over which rapid changes in material properties occur, and the macrocoordinate-the slow spatial 
variable-which corresponds to the typical length scale associated with the forcing functions of interest. The solution of a sequence of boundary-value problems defined over the unit cell yields the microstructure, and an appropriate projection of the equations dictated by their structure leads to the homogenized model which retains only the macrocoordinate as the independent variable.

This direct asymptotic technique yields a hierarchy of homogenized equations. For dynamic problems, the higher-order homogenized equations are of greater interest as they are capable of modeling the dominant phenomenon of dispersion. Unfortunately, there are a number of difficulties in using these dispersive models. Consequently, a variational approach (that utilizes the asymptotically derived microstructure) is used to construct the homogenized equations whose analysis is considerably simpler than that of the models derived by means of the direct method.

The multiscale technique for homogenization was first introduced by Keller [1], and its various aspects were considered by Babuska [2], Bensoussan et al. [3], Burgers [4], and Sanches-Palencia [5], among others. Many of these treatments were restricted to elliptic (static) problems and to the lowest-order homogenized models. Hyperbolic (dynamic) problems have been considered by Hegemier et al. [6], Maewal [7], and Santosa and Symes [8], the latter basing their approach on the expansion of the dispersion relation for Bloch waves. Here, we combine the multiscale technique with an orthogonal decomposition of the space of functions in which the differential equation is defined. This decomposition leads to a convenient method for the calculation of the microstructure to any desired degree of accuracy. Although the approach also leads directly to an asymptotically derived continuum model, we show that the variational scheme yields simpler homogenized equations.

The variational approach together with the introduction of the microcoordinate was used for jointed media by Murakami and Hegemier [9], who viewed the jointed medium essentially as a limiting case of a periodically laminated medium with alternating layers of two different materials, and the difficulty that they encountered in modeling the dispersion of waves propagating normal to the joints was alleviated by the introduction of a correction factor in a variationally derived constitutive law. Here we treat the joints $a b$ initio as planar surfaces of zero thickness and obtain a variationally derived continuum theory which does not require the introduction of any correction factors but nevertheless models the dispersion of normally incident waves quite accurately.

2. Formulation. We consider the one-dimensional deformation of a three-dimensional jointed medium. The joints are modeled as a single set of parallel, planar, periodically spaced surfaces, across which the displacements are allowed to be discontinuous. A typical joint in the one-dimensional domain shown in Fig. 1a is located at $2 n \bar{h}, n \in \mathscr{Z}$, with its two faces being identified by $2 n \bar{h}^{+}$and $2 n \bar{h}^{-}$. (Quantities

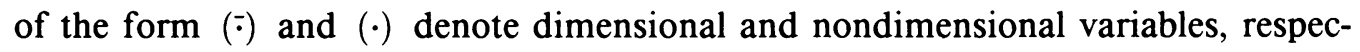
tively.) The governing relations for the displacement field $\bar{u}$ and the stress field $\bar{\sigma}$ are: 
(a) Equation of motion

$$
\frac{\partial \bar{\sigma}}{\partial \bar{x}}(\bar{x}, \bar{t})=\bar{\rho} \frac{\partial^{2} \bar{u}}{\partial \bar{t}^{2}}(\bar{x}, \bar{t})
$$

(b) Matrix constitutive law

$$
\bar{\sigma}(\bar{x}, \bar{t})=\bar{E} \frac{\partial \bar{u}}{\partial \bar{x}}(\bar{x}, \bar{t})
$$

(c) Stress continuity

$$
\bar{\sigma}\left(2 n \bar{h}^{-}, \bar{t}\right)=\bar{\sigma}\left(2 n \bar{h}^{+}, \bar{t}\right)
$$

(d) Joint constitutive law

$$
\bar{\sigma}\left(2 n \bar{h}^{-}, \bar{t}\right)=\bar{D}\left\{\bar{u}\left(2 n \bar{h}^{+}, \bar{t}\right)-\bar{u}\left(2 n \bar{h}^{-}, \bar{t}\right)\right\}
$$

where $\bar{D}$ is the tangent modulus for the joint.

For convenience, we nondimensionalize both the dependent and independent variables by using the following quantities:

$$
\begin{aligned}
& \bar{l} \quad \text { typical macro-dimension, } \\
& \bar{h} \quad \text { typical micro-dimension, } \\
& \bar{c}_{(m)}, \bar{\rho}_{(m)} \text { reference wave velocity and density, } \\
& \bar{t}_{(m)} \quad \text { typical macro signal travel time, } \\
& \bar{E}_{(m)} \quad \text { reference modulus. }
\end{aligned}
$$

The nondimensional quantities are then introduced according to:

$$
\begin{aligned}
& \bar{\sigma}=\bar{E}_{(m)} \sigma, \quad \bar{D}=\frac{\bar{E}_{(m)}}{\bar{h}} D, \quad \bar{E}=\bar{E}_{(m)} E, \\
& \bar{u}=\bar{l} u, \quad \bar{x}=\bar{l} x, \quad \bar{t}=\bar{t}_{(m)} t, \quad \bar{\rho}=\bar{\rho}_{(m)} \rho .
\end{aligned}
$$

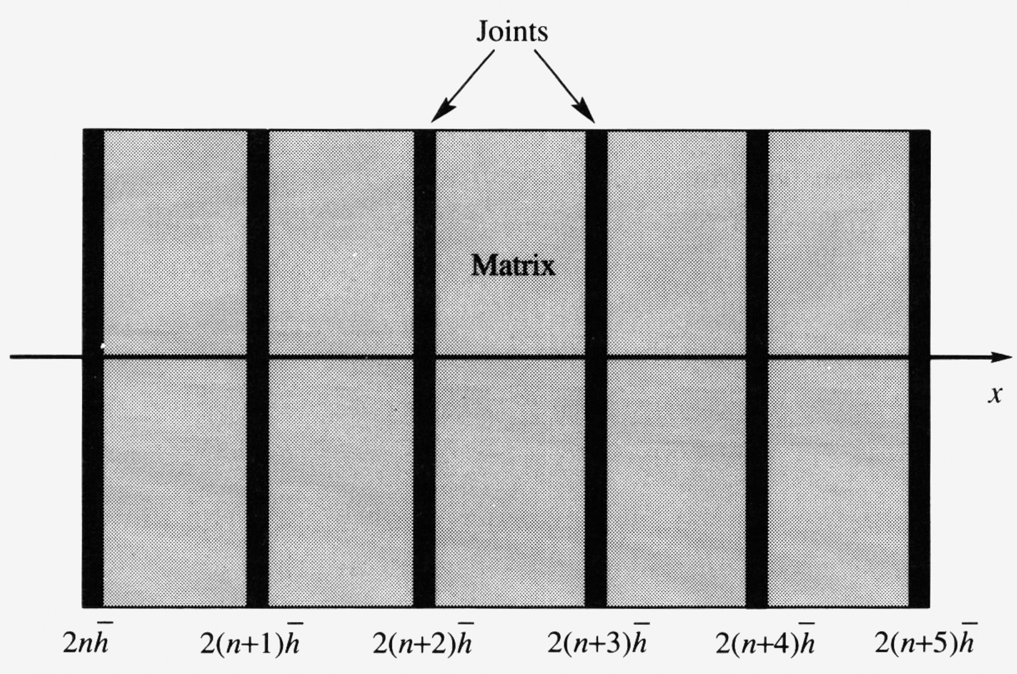

Fig. 1(a). Jointed medium 
With the scaled variables introduced as above, the nondimensional forms of the governing equations $(2.1)-(2.4)$ are obtained by simply dropping the overbars from the various quantities and by replacing $\bar{h}$ with $\bar{h} / \bar{l}$.

The statement of the initial boundary value problem is completed upon specification of boundary data and initial conditions. The class of initial conditions that is of interest is defined by

$$
u(x, 0)=f(x) \text { and } \frac{\partial u}{\partial t}(x, 0)=g(x),
$$

where the typical signal wavelengths associated with the functions $f$ and $g$ are much larger than the cell dimension. Therefore, there exists a minimum wavelength $\lambda_{\min }>\bar{h} / \bar{l}$ such that $f$ and $g$ are inverse Fourier transforms of functions $F$ and $G$ that have compact support in the wave number space: to wit

$$
[f, g](x)=\frac{1}{2 \pi} \int_{-2 \pi / \lambda_{\min }}^{2 \pi / \lambda_{\min }}[F, G](k) e^{i k x} d k
$$

Since our primary objective is the establishment of a continuum model valid away from the boundaries, the boundary conditions are not explicitly considered in the analysis.

We define $\varepsilon$, the ratio of micro-to-macro dimensions, as

$$
\varepsilon=\bar{h} / \bar{l} \ll 1 \text {. }
$$

With the variables defined as above, the joint interface conditions (2.2), (2.3) are seen to be periodic in the $x$-direction with period $2 \varepsilon$. Thus, the fields are expected to vary significantly over two length scales, suggesting the need for a multivariable asymptotic expansion procedure. A fast variable $\xi$ and slow variable $x^{*}$ (the two being independent) are introduced according to

$$
\xi=\phi^{-1}(\varepsilon) x, \quad \phi(\varepsilon) \rightarrow 0 \quad \text { as } \varepsilon \rightarrow 0
$$

and

$$
x^{*}=\psi(\varepsilon) x, \quad \psi(\varepsilon) \rightarrow 1 \quad \text { as } \varepsilon \rightarrow 0 .
$$

For our analysis we use

$$
\phi(\varepsilon)=\varepsilon \quad \text { and } \quad \psi(\varepsilon)=1 .
$$

All field variables are now functions of the microcoordinate $\xi$ as well as the macrocoordinate $x^{*}$, so that for any field $f$,

$$
f(x, t ; \varepsilon)=f^{*}\left(x^{*}, \xi, t ; \varepsilon\right)
$$

and the spatial derivatives take the form

$$
\frac{\partial f}{\partial x}=\frac{\partial f^{*}}{\partial x}+\frac{1}{\varepsilon} \frac{\partial f^{*}}{\partial \xi}
$$

With the introduction of the fast spatial coordinate $\xi$, the joints are seen to be located at $\xi=2 n \bar{h} /(\bar{l} \varepsilon) \equiv 2 n h$. The field variables are thus periodic functions of $\xi$, with the length of periodicity being $2 h$, the joint spacing. It is, therefore, necessary to treat only a typical unit cell associated with the $\xi$-direction. With no loss of 
generality, the cell may be defined by $-h<\xi \leq h$, with the joints located at the cell boundaries. (Note, however, that only one of the joints at $\xi=h$ is included in the unit cell.)

With the fields now being functions of both $\xi$ and $x$ (for convenience we replace $(\cdot)^{*}$ with $(\cdot)$ in all subsequent analysis), the boundary value problem can be stated as

(a) Equation of motion

$$
\frac{\partial \sigma}{\partial x}+\frac{1}{\varepsilon} \frac{\partial \sigma}{\partial \xi}=\rho \frac{\partial^{2} u}{\partial t^{2}}
$$

and

(b) Matrix constitutive law

$$
\sigma=E\left(\frac{\partial u}{\partial x}+\frac{1}{\varepsilon} \frac{\partial u}{\partial \xi}\right) .
$$

With the aforementioned choice of the unit cell (Fig. 1b), the periodicity conditions are given by

$$
\begin{aligned}
& \sigma\left(x, h^{+}, t ; \varepsilon\right)=\sigma\left(x,-h^{+}, t ; \varepsilon\right), \\
& u\left(x, h^{+}, t ; \varepsilon\right)=u\left(x,-h^{+}, t ; \varepsilon\right) .
\end{aligned}
$$

Here the two faces of the joint at $\xi=h$ within the unit cell are denoted by $h^{+}$and $h^{-}$.

The periodicity conditions $(2.12 \mathrm{a}, \mathrm{b})$ together with the interface conditions $(2.3)$ written as

$$
\sigma\left(x, h^{+}, t ; \varepsilon\right)=\sigma\left(x, h^{-}, t ; \varepsilon\right)=\frac{D}{\varepsilon}\left[u\left(x, h^{+}, t ; \varepsilon\right)-u\left(x, h^{-}, t ; \varepsilon\right)\right]
$$

lead to the following:

(c) Stress continuity

$$
\sigma(x, h, t ; \varepsilon)=\sigma(x,-h, t ; \varepsilon)
$$

and

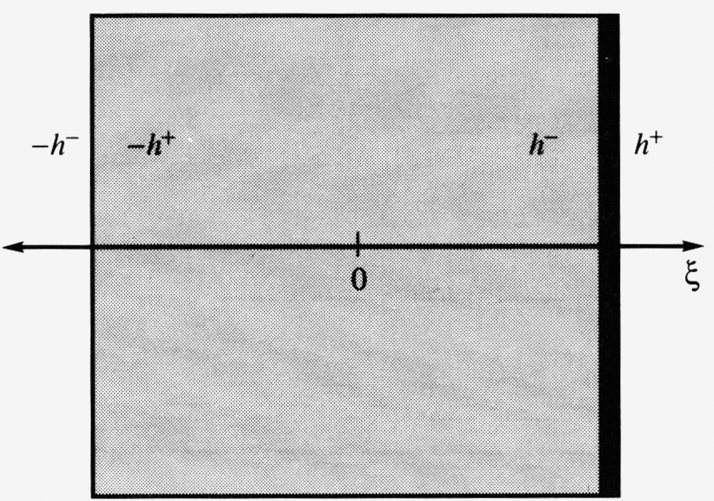

FIG. 1(b). Unit cell 
(d) Joint constitutive law

$$
\sigma(x, h, t ; \varepsilon)=\frac{D}{\varepsilon}[u(x,-h, t ; \varepsilon)-u(x, h, t ; \varepsilon)] .
$$

For convenience, we have replaced $h^{-}$by $h$ and $-h^{+}$by $-h$ in (2.14), (2.15), since no reference is made to the values of the field variables at $\xi=h^{+}$in the following sections.

\section{Analysis.}

3.1. Introductory remarks. Combining Eqs. (2.10) and (2.11) we obtain

$$
M u(x, \xi, t ; \varepsilon) \equiv \frac{1}{\varepsilon^{2}} \frac{\partial^{2} u}{\partial \xi^{2}}+\frac{2}{\varepsilon} \frac{\partial^{2} u}{\partial \xi \partial x}+\frac{\partial^{2} u}{\partial x^{2}}-\frac{1}{c^{2}} \frac{\partial^{2} u}{\partial t^{2}}=0
$$

This equation is the basis of the subsequent development wherein we construct a continuum model of the jointed medium by means of an asymptotic scheme and eventual elimination of the microcoordinate $\xi$. In this process the terms $\frac{\partial^{n} u}{\partial x^{n}}$ and $\frac{\partial^{n} u}{\partial t^{n}}$ are considered to be $O(1)$ for all $n$ so that the order of each term in Eq. (3.1) is strictly a function of its $\varepsilon$-dependent coefficient. This result follows from the fact that the spatial derivatives of $u$ are of $O\left(k^{n}\right)$ in wave number space (see Eq. (2.6)). Since we have restricted our consideration to initial disturbances with wavenumbers bounded from above by unity in the scaled system, variables of $O\left(k^{n}\right)$ will always be of order unity. As for the order of the temporal derivatives of $u$, we can consider their Fourier components in the frequency domain. An analysis similar to Santosa and Symes's [8, Theorem 1] leads to the conclusion that the contribution of all modes but the lowest, the so-called acoustic mode, is negligible whenever $\varepsilon \ll 1$. Since for the acoustic mode, the order of the frequency is (for small wave numbers) the same as that of the wavenumber and, therefore, unity, it is evident that the temporal derivatives of $u$ are also $O(1)$.

The differential equation (3.1) with the boundary terms

$$
\begin{gathered}
\frac{1}{\varepsilon} \frac{\partial u}{\partial \xi}(-h)=-\frac{\partial u}{\partial x}(-h)+\frac{1}{\varepsilon} D s, \\
\frac{1}{\varepsilon} \frac{\partial u}{\partial \xi}(h)=-\frac{\partial u}{\partial x}(h)+\frac{1}{\varepsilon} D s, \\
s \equiv u(-h)-u(h)
\end{gathered}
$$

is defined in the real Hilbert space $L^{2}(\Omega)$ where $\Omega=[-l, l] \times[-h, h] \times\left[t_{0}, t_{1}\right]$. Hence $M u \in L^{2}(\Omega)$ must then be square integrable over the unit cell by a simple application of Fubini's Theorem. In this section we restrict our attention to the closed linear subspace $S_{1} \equiv L^{2}[-h, h]$, with the inner product

$$
(a, b)_{S_{1}} \equiv \frac{1}{2 h} \int_{-h}^{h} a(\xi) b(\xi) d \xi
$$

and analyze the operator strictly in this subspace. We define $L \equiv \partial^{2} / \partial \xi^{2}$ as the leading operator of $M$ in (3.1) and use its properties under periodic boundary conditions to project any vector $k \in S_{1}$ into two orthogonal spaces in $S_{1}$. This decomposition 
of the vector leads directly to two distinct differential equations. The first is solved to obtain the microstructure via an asymptotic scheme, while the second equation yields the continuum model.

3.2. Development. With $L u(\xi, x, t, \varepsilon) \in S_{1}$, the leading boundary terms in (3.2) are homogeneous and are written as $B u=0$, where

$$
B u \equiv\left[B_{1} u B_{2} u\right] \text { and } B_{1} u \equiv \frac{\partial u}{\partial \xi}(-h)-D s, B_{2} u \equiv \frac{\partial u}{\partial \xi}(h)-D s .
$$

We make the key observation that the operator $L$ is singular, possessing a nontrivial kernel spanned by the constant function 1 , and (as we show later in this section) is selfadjoint. With this in mind we state the following well-known theorem:

Theorem 3.1. Let $M$ be a closed subspace of a Hilbert space $H$. Then every $x \in H$ has a unique orthogonal decomposition $x=P x+Q x$ into a sum of $P x \in M$ and $Q x \in M^{\perp}$, where $M^{\perp}=\{y \in H: y \perp x\}$ (see [11] for a simple proof).

CoRollary 3.1. Any vector $k \in S_{1}$ can be uniquely expressed by the orthogonal decomposition $k=k_{r}+k_{n}$, where $\left(k_{r}, k_{n}\right)_{S_{1}}=0 ; k_{r}$ is in the range of $L, R(L)$; and $k_{n}$ is in its null space, $N(L)$.

Proof. $N\left(L^{*}\right)=N(L)$ is a closed linear subspace of $S_{1}$ since it is spanned by the constant functions. Let $f \in R(L), v \in N\left(L^{*}\right)$, and $u$ be in the domain of $L$. Since $(v, f)_{S_{1}}=(v, L u)_{S_{1}}=\left(u, L^{*} v\right)_{S_{1}}=0$, it follows that $N(L) \perp R(L)$. Application of Theorem 3.1 completes the proof.

By applying Corollary 3.1 to the vector $M u(\xi, x, t ; \varepsilon) \in S_{1}$ and noting that $M u=0$ (and hence its projection into any subspace), we obtain the following two differential equations:

$$
\begin{gathered}
(M u)_{n} \equiv(M u, 1)_{S_{1}} \cdot 1=0, \\
(M u)_{r} \equiv M u-(M u, 1)_{S_{1}} \cdot 1=0 .
\end{gathered}
$$

The above decomposition leads to a formulation of the microstructure problem which has a unique solution even though it has a singular leading operator. This issue will be more thoroughly addressed in the later part of this section.

Written out explicitly, Eq. (3.8) takes the form

$$
\begin{aligned}
\frac{1}{\varepsilon^{2}} \frac{\partial^{2} u}{\partial \xi^{2}} & +\frac{2}{\varepsilon} \frac{\partial^{2} u}{\partial \xi \partial x}+\frac{\partial^{2} u}{\partial x^{2}}-\frac{1}{c^{2}} \frac{\partial^{2} u}{\partial t^{2}} \\
& -\frac{1}{2 h} \int_{-h}^{h}\left(\frac{1}{\varepsilon^{2}} \frac{\partial^{2} u}{\partial \xi^{2}}+\frac{2}{\varepsilon} \frac{\partial^{2} u}{\partial \xi \partial x}+\frac{\partial^{2} u}{\partial x^{2}}-\frac{1}{c^{2}} \frac{\partial^{2} u}{\partial t^{2}}\right) d \xi=0
\end{aligned}
$$

and Eq. (3.7) yields

$$
\frac{1}{2 h} \int_{-h}^{h}\left(\frac{1}{\varepsilon^{2}} \frac{\partial^{2} u}{\partial \xi^{2}}+\frac{2}{\varepsilon} \frac{\partial^{2} u}{\partial \xi \partial x}+\frac{\partial^{2} u}{\partial x^{2}}-\frac{1}{c^{2}} \frac{\partial^{2} u}{\partial t^{2}}\right) d \xi=0
$$

or, equivalently,

$$
\frac{1}{2 h} \int_{-h}^{h}\left(\frac{\partial \sigma}{\partial x}-\rho \frac{\partial^{2} u}{\partial t^{2}}\right) d \xi=0
$$


By combining (3.10) with the stress continuity condition $\left[\frac{1}{\varepsilon} \frac{\partial u}{\partial \xi}+\frac{\partial u}{\partial x}\right]_{-h}^{h}=0$, we obtain $\frac{1}{\varepsilon^{2}} \frac{\partial^{2} u}{\partial \xi^{2}}+\frac{2}{\varepsilon} \frac{\partial^{2} u}{\partial \xi \partial x}+\frac{\partial^{2} u}{\partial x^{2}}-\frac{1}{c^{2}} \frac{\partial^{2} u}{\partial t^{2}}-\left.\frac{1}{2 h} \frac{1}{\varepsilon} \frac{\partial u}{\partial x}\right|_{-h} ^{h}-\frac{1}{2 h} \int_{-h}^{h}\left(\frac{\partial^{2} u}{\partial x^{2}}-\frac{1}{c^{2}} \frac{\partial^{2} u}{\partial t^{2}}\right) d \xi=0$.

This equation is solved asymptotically for the microstructure by another application of Corollary 3.1 and the decomposition of the solution as

$$
u=u_{0}+w \quad \text { where } u_{0} \in N(L) .
$$

We impose the condition

$$
w(x, 0, t ; \varepsilon)=0
$$

which renders $u_{0}$ to be the cell-centered displacement ${ }^{1}$

$$
u_{0}=u(x, 0, t ; \varepsilon) .
$$

3.3. Asymptotic scheme. If the ansatz (3.12) is substituted into (3.2) and (3.11), we obtain a boundary value problem for $w$ whose solution is dependent on both $\varepsilon$ and $u_{0}$ :

$$
\frac{\partial^{2} w}{\partial \xi^{2}}=\varepsilon b(x, \xi, t ; \varepsilon)
$$

and

$$
\begin{gathered}
\frac{\partial w}{\partial \xi}(x, \pm h, t ; \varepsilon)-D(w(x,-h, t ; \varepsilon)-w(x, h, t, \varepsilon)) \\
=-\varepsilon\left(\frac{\partial u_{0}}{\partial x}(x, \pm h, t ; \varepsilon)+\frac{\partial w}{\partial x}(x, \pm h, t ; \varepsilon)\right)
\end{gathered}
$$

where

$$
\begin{aligned}
b(x, \xi, t ; \varepsilon) \equiv & -2 \frac{\partial^{2} w}{\partial \xi \partial x}-\varepsilon \frac{\partial^{2} w}{\partial x^{2}}+\frac{\varepsilon}{c} \frac{\partial^{2} w}{\partial t^{2}}+\left.\frac{1}{2 h} \frac{\partial w}{\partial x}\right|_{-h} ^{h} \\
& +\varepsilon \frac{1}{2 h} \int_{-h}^{h}\left(\frac{\partial^{2} w}{\partial x^{2}}-\frac{1}{c^{2}} \frac{\partial^{2} w}{\partial t^{2}}\right) d \xi
\end{aligned}
$$

From Theorem 3.2, we may invert the leading operator of (3.15) with boundary conditions (3.16) so that we may reduce the boundary-value problem to the following equation:

$$
H\left(w, u_{0}, \varepsilon\right)=w-\varepsilon G b+\left.\varepsilon G_{1}\left(\frac{\partial u_{0}}{\partial x}+\frac{\partial w}{\partial x}\right)\right|_{h}+\left.\varepsilon G_{2}\left(\frac{\partial u_{0}}{\partial x}+\frac{\partial w}{\partial x}\right)\right|_{-h}-\varepsilon \beta=0 .
$$

For $\varepsilon=0$ and any $u_{0}$, the solution of this problem is $w=0$ because of the uniqueness condition (3.13). By considering the Fréchet derivative $H^{\prime}$ of $H$ with respect to $w$, we see that there is a neighborhood of $\varepsilon=0$ and any arbitrary but fixed $u_{0}$ where $H^{\prime}$ is nonsingular. Therefore, by the Implicit Function Theorem,

\footnotetext{
${ }^{1}$ Alternatively, it is also possible to impose $(w, 1)_{S_{1}}=0$, so that $u_{0}=(u, 1)_{S_{1}}$ and is thereby the cell-averaged displacement.
} 
$w$ may be written as a continuous function $q$ of $\varepsilon$ and $u_{0}$ in this neighborhood. Obviously, the smoothness and analyticity of $q$ depends on that of $H$ and the size of the neighborhood is determined by the required order of the continuum model. In this neighborhood, the solution may take the form of a convergent power series with the number of terms again dictated by the desired order of the continuum model. Moreover, since $u_{0}$ is fixed for the expansion of $w$ about $\varepsilon=0$, we may consider $u_{0}$ to be a parameter for the computation of $w$, i.e., for this purpose, $\varepsilon^{n} \partial_{x}^{k} \partial_{t}^{l} u_{0}=O\left(\varepsilon^{n}\right) \forall k, l, n \in \mathscr{Z}^{+}$.

Thus the solution to Eq. (3.11) is obtained by expanding the dependent variable $w$ of (3.12) in the form of the asymptotic series

$$
w(x, \xi, t ; \varepsilon)=\sum_{n=1}^{N} \varepsilon^{n} u^{(n)}(x, \xi, t),
$$

where $u^{(n)}, n \in \mathcal{N}$, is required to satisfy the uniqueness condition of the previous section. Substituting Eqs. (3.12) and (3.17) into (3.2) and (3.11) results in the sequence of differential equations

$$
\begin{gathered}
\frac{\partial^{2} u^{(n)}}{\partial \xi^{2}}+2 \frac{\partial^{2} u^{(n-1)}}{\partial \xi \partial x}+\frac{\partial^{2} u^{(n-2)}}{\partial x^{2}}-\frac{1}{c^{2}} \frac{\partial^{2} u^{(n-2)}}{\partial t^{2}}-\left.\frac{1}{2 h} \frac{\partial u^{(n-1)}}{\partial x}\right|_{-h} ^{h} \\
-\frac{1}{2 h} \int_{-h}^{h}\left(\frac{\partial^{2} u^{(n-2)}}{\partial x^{2}}-\frac{1}{c^{2}} \frac{\partial^{2} u^{(n-2)}}{\partial t^{2}}\right) d \xi=0
\end{gathered}
$$

and the following sequence of boundary conditions (note that stress continuity has been ensured):

$$
\frac{\partial u^{(n)}}{\partial \xi}( \pm h)+\frac{\partial u^{(n-1)}}{\partial x}( \pm h)=D\left[u^{(n)}(-h)-u^{(n)}(h)\right] .
$$

Here $n \in \mathscr{N}, u^{(0)} \equiv u_{0}$, and $u^{(-1)} \equiv 0$.

We now show that the sequence of problems for the calculation of the microstructure as formulated in (3.13), (3.18), (3.19) have unique solutions in the Sobolev space $H^{1}(-h, h)$ where $H^{m}$ is defined as the completion of $C^{\infty}(-h, h)$, with respect to $\|\cdot\|_{H^{m}(-h, h)}$, and

$$
\|u\|_{H^{m}(-h, h)}^{2}=\sum_{\alpha \leq m}\left\|D^{\alpha} u\right\|_{S_{1}}^{2} .
$$

To this end, we write the MBVP (3.18) with boundary conditions (3.19) as

$$
\begin{gathered}
L u^{(n)}(\xi ; x, t, \varepsilon)=f(\xi ; x, t, \varepsilon), \quad-h<\xi<h ; \\
\frac{\partial u^{(n)}}{\partial \xi}(-h ; x, t, \varepsilon)-D s^{(n)}=k_{1}, \\
\frac{\partial u^{(n)}}{\partial \xi}(h ; x, t, \varepsilon)-D s^{(n)}=k_{2} .
\end{gathered}
$$


For the purposes of the subsequent analysis we drop the superscript from $u^{(n)}$ and $s^{(n)}$ and do not explicitly write the dependence of the field variables on the parameters. We require $f \in L^{2}(-h, h)$ (this is satisfied since $(x, t, \varepsilon) \rightarrow u_{0}(\cdot ; x, t, \varepsilon)$ is a constant) and note that $k_{1}, k_{2}$ are constants. We define the space $W$ as the completion of $C^{\infty}$-functions that satisfy (3.22) with homogeneous boundary data.

Noting that $H^{1}(-h, h)$ is densely embedded in $L^{2}(-h, h)$, it is easy to see that when $u, v \in W$, the operator $L$ satisfies the condition $(L u, v)_{L^{2}(-h, h)}=$ $(u, L v)_{L^{2}(-h, h)}$ and hence is selfadjoint. When considering the inhomogeneous boundary data, however, we see that for $u \in H^{m}(-h, h)$ the bilinear concomitant of $L$ does not vanish and we obtain

$$
(L u, v)_{L^{2}(-h, h)}=\left(u, L^{*} v\right)_{L^{2}(-h, h)}-\left\langle u,\left.v\right|_{-h} ^{h} \quad \forall u \in H^{m}(-h, h), \forall v \in W .\right.
$$

By defining $\widehat{L}$ as the operator satisfying $(\widehat{L} u, v)_{L^{2}(-h, h)}=\left(u, L^{*} v\right)_{L^{2}(-h, h)}$ we find that

$$
\widehat{L} u=\frac{\partial^{2} u}{\partial \xi^{2}}+\left[\frac{\partial u}{\partial \xi}(-h)-D s\right] \delta_{-h}-\left[\frac{\partial u}{\partial \xi}(h)-D s\right] \delta_{h},
$$

which is the form the dual of $H^{1}(-h, h)$ must take (see [12]). Hence, the generalization of (3.21) becomes

$$
\widehat{L} u=\hat{f},
$$

where

$$
\hat{f}=f+k_{1} \delta_{-h}-k_{2} \delta_{h}
$$

LEMMA 3.1. The solvability condition for (3.23) is $\int_{-h}^{h} f d \xi=k_{2}-k_{1}$.

Proof. We require $\hat{f}$ to be in the range of $\hat{L}$, which is the orthogonal complement of the null space of $\widehat{L}$. Since the one-dimensional null space of $\widehat{L}$ is spanned by the constant function 1 , a solution exists to $(3.23)$ if $(\hat{f}, 1)_{L^{2}(-h, h)}=0$ as dictated by the Fredholm Alternative Theorem.

Theorem 3.2. There exists $u \in H^{1}(-h, h)$ satisfying (3.23) which takes the form

$$
u=G f+G_{1} k_{1}+G_{2} k_{2}+\beta \text {. }
$$

Proof. To ensure the satisfaction of Lemma 3.1, we solve

$$
\widehat{L} u=P_{R(L)} \hat{f} \text {, }
$$

where the projection of $\hat{f}$ into the range of $L$ takes the following form:

$$
P_{R(L)} \hat{f}=\hat{f}-(\hat{f}, 1)_{S_{1}} \cdot 1 .
$$

Written out explicitly, we solve

$$
\frac{\partial^{2} u}{\partial \xi^{2}}=f-\frac{1}{2 h}\left[k_{1}-k_{2}+\int_{-h}^{h} f d s\right] \text {. }
$$

Integration by parts and application of the boundary conditions leads to the solution in the form of a generalized Green's function:

$$
u(\xi)=\int_{-h}^{h} g(\xi, s) f(s) d s+\left(-\frac{\xi^{2}}{4 h}+\frac{\xi}{2 \Gamma}\right) k_{1}+\left(\frac{\xi^{2}}{4 h}+\frac{\xi}{2 \Gamma}\right) k_{2}+\beta,
$$


where

$$
g(\xi, s)=H(\xi-s)(\xi-s)-\frac{\xi^{2}}{4 h}-\frac{\frac{1}{2}+D(h-s)}{1+2 D h} \xi,
$$

and $H$ is the Heaviside distribution. With $f \in L^{2}(-h, h)$ and a continuous function being bounded on a compact set, an application of the Dominated Convergence Theorem verifies that $u \in H^{1}(-h, h)$.

Corollary 3.2. The solution to $(3.18),(3.19)$ with $u^{(n)}(0 ; x, t, \varepsilon)=0$ exists and is unique $\forall n \in \mathscr{N}$.

Proof. With the functions $f, k_{1}$, and $k_{2}$ in (3.21), (3.22) identified by (3.18) and (3.19), a simple integration verifies that the solvability condition of Lemma 3.1 is identically satisfied. Therefore, the solution exists and is given by (3.24), with $\beta$ obtained as

$$
\beta=-\int_{-h}^{h} g(0, s) f(s) d(s) .
$$

We may now consider the series of microstructure boundary-value problems (termed MBVPs) for each order of $\varepsilon$ obtained from the asymptotic expansions. The first of these, which is $O\left(\frac{1}{\varepsilon}\right)$, turns out to be

$$
\frac{\partial^{2} u^{(1)}}{\partial \xi^{2}}+2 \frac{\partial^{2} u^{(0)}}{\partial \xi \partial x}-\left.\frac{1}{2 h} \frac{\partial u^{(0)}}{2 x}\right|_{-h} ^{h}=0
$$

with

$$
\frac{\partial u^{(0)}}{\partial x}(h)+\frac{\partial u^{(1)}}{\partial \xi}(h)=D\left[u^{(1)}(-h)-u^{(1)}(h)\right] .
$$

Applying Theorem 3.2 and Corollary 3.2, we obtain

$$
u^{(1)}(x, \xi, t)=-\frac{1}{\Gamma} \frac{\partial u^{(0)}}{\partial x} \xi, \quad \text { where } \Gamma=1+2 D h .
$$

In a similar fashion, we obtain the following solutions up to $O\left(\varepsilon^{2}\right)$ (i.e., up to $\left.u^{(4)}\right)$ :

$$
\begin{aligned}
& u^{(2)}=\frac{1}{2 \Gamma} \frac{\partial^{2} u^{(0)}}{\partial x^{2}} \xi^{2}, \\
& u^{(3)}=-\frac{1}{6 \Gamma}\left(\frac{\partial^{3} u^{(0)}}{\partial x^{3}}+\frac{1}{c^{2}} \frac{\partial^{3} u^{(0)}}{\partial t^{2} \partial x}\right) \xi^{3}+\left(\gamma \frac{\partial^{3} u^{(0)}}{\partial x^{3}}+\delta \frac{1}{c^{2}} \frac{\partial^{3} u^{(0)}}{\partial t^{2} \partial x}\right) \xi, \\
& u^{(4)}=\frac{1}{24 \Gamma}\left(\frac{\partial^{4} u^{(0)}}{\partial x^{4}}+\frac{3}{c^{2}} \frac{\partial^{4} u^{(0)}}{\partial t^{2} \partial x^{2}}\right) \xi^{4}-\frac{1}{2}\left(\gamma \frac{\partial^{4} u^{(0)}}{\partial x^{4}}+\frac{\alpha}{c^{2}} \frac{\partial^{4} u^{(0)}}{\partial t^{2} \partial x^{2}}\right) \xi^{2},
\end{aligned}
$$

where we have used the definitions

$$
\gamma=\frac{2 D h^{3}}{6 \Gamma^{2}}, \quad \delta=\frac{3 h^{2}+2 D h^{3}}{6 \Gamma^{2}}, \quad \alpha=\frac{5 h^{2}+6 D h^{3}}{6 \Gamma^{2}} .
$$

The equations (2.11) and (3.10), (3.12), (3.17) lead to the asymptotically derived continuum model

$$
\frac{2 D h}{\Gamma} \frac{\partial^{2} u^{(0)}}{\partial x^{2}}+\varepsilon^{2} \frac{D h^{3}}{3 \Gamma^{2}} \frac{\partial^{4} u^{(0)}}{\partial x^{4}}+\frac{\varepsilon^{2}}{c^{2}}\left(\frac{3 h^{2}+2 D h^{3}}{6 \Gamma^{2}}-\frac{2 h^{3}}{6 \Gamma}\right) \frac{\partial^{4} u^{(0)}}{\partial t^{2} \partial x^{2}}-\rho \frac{\partial^{2} u^{(0)}}{\partial t^{2}}=0 .
$$


Note that terms up to $u^{(3)}$ have been used in the construction of (3.30) which is the lowest-order continuum theory capable of modeling dispersive effects.

At least in principle, the solution of this differential equation together with appropriate boundary and initial data, is expected to yield an asymptotically exact solution of the original problem (2.1)-(2.4). However, it is evident that in order to pose the initial boundary-value problem, one must be able to prescribe the boundary conditions required for a solution. Whereas for the zeroth-order homogenization it is easy to show that $u \rightarrow u^{(0)}$ weakly in $H^{1}$ and thus boundary data are easily determined, for higher-order homogenization such issues are very difficult to resolve. For this reason, we make use of a mixed variational principle which simultaneously alleviates the problem of the possible loss of ellipticity of the purely spatial part of the homogenized operator (3.30). The trial functions used in the variational approach span the space of solutions to the microstructure problems.

4. Variational procedure. By viewing the boundary-value problem in a distributional (or weak) sense, we obtain a functional, which upon extremization yields equations describing the behavior of the continuum along with the proper boundary conditions necessary for the solutions.

Consider the following functional:

$$
J=\int_{-l}^{l}\left\{\int_{-h}^{h}\left[\sigma\left(\frac{\partial u}{\partial x}+\frac{1}{\varepsilon} \frac{\partial u}{\partial \xi}\right)-\frac{\sigma^{2}}{2 E}\right] d \xi+\frac{1}{\varepsilon}[u(-h)-u(h)] \sigma(h)-\frac{\sigma(h)^{2}}{2 D}\right\} d x,
$$

where the displacement field $u$ and the stress field $\sigma$ are subjected to independent variation. By setting

$$
\delta J+\int_{-l}^{l} \int_{-h}^{h} \rho \frac{\partial^{2} u}{\partial t^{2}} \delta u d \xi d x=0
$$

we obtain the variationally equivalent boundary value problem (2.10)-(2.15).

The trial functions for the displacement and stress fields are obtained from the asymptotic analysis of the previous section. They are

$$
u(x, \xi, t ; \varepsilon)=u_{0}(x, t)+\varepsilon \psi(x, t) \xi+\varepsilon^{2} \phi(x, t) f(\xi)
$$

and

$$
\sigma(x, \xi, t ; \varepsilon)=\sigma_{0}(x, t)+\varepsilon^{2} Q(x, t) f(\xi)+\varepsilon^{3} R(x, t) g(\xi)
$$

where $f(\xi)$ and $g(\xi)$ are defined as

$$
f(\xi) \equiv \xi^{2}-\frac{1}{3} h^{2}, \quad g(\xi) \equiv \xi^{3}-h^{2} \xi .
$$

Here $\psi$ and $\phi$ play the role of higher-order (generalized) displacement fields and $Q$ and $R$ play the role of higher-order (generalized) stresses. (Note that with the preceding choice of the basis functions, $u_{0}$ and $\sigma_{0}$ are the cell-averaged displacement and stress, repectively.) It is evident that the stress trial function is of higher order than the displacement trial function. The $O\left(\varepsilon^{3}\right)$ term was added due to the absence of the $O(\varepsilon)$ term in the stress microstructure field obtained by the asymptotic analysis of 
the previous section. Without the addition of this higher-order $\varepsilon^{3}$ term, a quadratic stress field was found to yield spurious zero energy modes in the continuum model.

By substituting these fields into Eq. (4.2), we obtain

$$
\begin{aligned}
\delta \int_{-l}^{l}\left\{\int _ { - h } ^ { h } \left\{\left[\sigma_{0}+\varepsilon^{2} Q f(\xi)+\varepsilon^{3} R g(\xi)\right]\left[\frac{\partial u_{0}}{\partial x}+\varepsilon \frac{\partial \psi}{\partial x} \xi+\varepsilon^{2} \frac{\partial \phi}{\partial x} f(\xi)\right]\right.\right. \\
+\left[\sigma_{0}+\varepsilon^{2} Q f(\xi)+\varepsilon^{3} R g(\xi)\right][\psi+2 \varepsilon \phi \xi] \\
\left.-\frac{1}{2 E}\left[\sigma_{0}^{2}+\varepsilon^{4} Q^{2} f(\xi)^{2}+\varepsilon^{6} R^{2} g(\xi)^{2}\right]\right\} d \xi \\
\left.-2 \psi h\left[\sigma_{0}+\frac{2}{3} \varepsilon^{2} h^{2} Q\right]-\frac{1}{2 D}\left[\sigma_{0}^{2}+\frac{4}{3} \varepsilon^{2} h^{2} \sigma_{0} Q+\frac{4}{9} \varepsilon^{4} h^{4} Q^{2}\right]\right\} d x \\
=\int_{-l}^{l} \int_{-h}^{h}\left[\rho \frac{\partial^{2} u_{0}}{\partial t^{2}} \delta u_{0}+\varepsilon^{2} \rho \frac{\partial^{2} \psi}{\partial t^{2}} \delta \psi \xi^{2}+\varepsilon^{4} \rho \frac{\partial^{2} \phi}{\partial t^{2}} \delta \phi f(\xi)^{2}\right] d \xi d x .
\end{aligned}
$$

Integration over the microstructural domain yields

$$
\begin{aligned}
\int_{-l}^{l}\left\{\sigma_{0} \delta \frac{\partial u_{0}}{\partial x}+\frac{\partial u_{0}}{\partial x} \delta \sigma_{0}+\frac{4 \varepsilon^{4} h^{4}}{45}\left(Q \delta \frac{\partial \phi}{\partial x}+\frac{\partial \phi}{\partial x} \delta Q\right)\right. \\
-\frac{2 \varepsilon^{4} h^{4}}{15}\left(R \delta \frac{\partial \psi}{\partial x}+\frac{\partial \psi}{\partial x} \delta R\right)-\frac{4 \varepsilon^{4} h^{4}}{15}(R \delta \phi+\phi \delta R) \\
-\frac{\sigma_{0}}{E} \delta \sigma_{0}-\frac{4 \varepsilon^{4} h^{4}}{45 E} Q \delta Q-\frac{8 \varepsilon^{6} h^{6}}{105 E} R \delta R-\frac{2 \varepsilon^{2} h^{2}}{3}(Q \delta \psi+\psi \delta Q) \\
-\frac{\sigma_{0}}{2 D h} \delta \sigma_{0}-\frac{\varepsilon^{2} h^{2}}{3 D h}\left(\sigma_{0} \delta Q+Q \delta \sigma_{0}\right)-\frac{2 \varepsilon^{4} h^{4}}{9 D h} Q \delta Q \\
\left.+\rho \frac{\partial^{2} u_{0}}{\partial t^{2}} \delta u_{0}+\frac{1}{3} \rho \varepsilon^{2} h^{2} \frac{\partial^{2} \psi}{\partial t^{2}} \delta \psi+\frac{4 \varepsilon^{4} h^{4}}{45} \rho \frac{\partial^{2} \phi}{\partial t^{2}} \delta \phi\right\} d x=0
\end{aligned}
$$

Rerdering the variations arbitrary, we obtain the following Euler-Lagrange equations:

$$
\begin{gathered}
\frac{\partial \sigma_{0}}{\partial x}=\rho \frac{\partial^{2} u_{0}}{\partial t^{2}}, \\
-\frac{2}{5} \frac{\partial \bar{R}}{\partial x}+\frac{2}{\varepsilon^{2} h^{2}} \bar{Q}=\rho \frac{\partial^{2} \psi}{\partial t^{2}}, \\
\frac{1}{\varepsilon^{2} h^{2}} \frac{\partial \bar{Q}}{\partial x}+\frac{3}{\varepsilon^{2} h^{2}} \bar{R}=\rho \frac{\partial^{2} \phi}{\partial t^{2}} ; \\
\frac{\partial u_{0}}{\partial x}=\left(\frac{1}{E}+\frac{1}{2 D h}\right) \sigma_{0}+\frac{1}{3 D h} \bar{Q}, \\
\frac{4 \varepsilon^{2} h^{2}}{45} \frac{\partial \phi}{\partial x}-\frac{2}{3} \psi=\frac{1}{3 D h} \sigma_{0}+\left(\frac{4}{45 E}+\frac{2}{9 D h}\right) \bar{Q}, \\
-\left(\frac{\partial \psi}{\partial x}+2 \phi\right)=\frac{4}{7 E} \bar{R},
\end{gathered}
$$

where $\bar{R}=R \varepsilon^{2} h^{2}$ and $\bar{Q}=Q \varepsilon^{2} h^{2}$. 
Equations (4.8a, b, c) are the homogenized equations of motion, and Eqs. (4.9a, b, c) are the stress-displacement relations for the continuum.

On the boundary we must prescribe the quantities

$$
\begin{gathered}
\sigma_{0} \text { or } u_{0}, \\
\bar{Q} \text { or } \phi, \\
\bar{R} \text { or } \psi .
\end{gathered}
$$

With regard to the question of appropriate initial conditions to be used with this theory, since only the second derivative with respect to time appears in the EulerLagrange equations, as in the wave equation, it is appropriate to prescribe at time $t=0$

$$
\begin{gathered}
u_{0} \text { and } \frac{\partial u_{0}}{\partial t} \\
\psi \text { and } \frac{\partial \psi}{\partial t} \\
\phi \text { and } \frac{\partial \phi}{\partial t}
\end{gathered}
$$

5. Dispersion. In an attempt to test the efficacy of the continuum models, the phase velocity spectra of the (1) asymptotically and (2) variationally derived theories have been compared with the exact results (see Appendix) for time harmonic waves propagating through the one-dimensional medium considered here.

(1) By substituting $u_{0}=U e^{i(k x-\omega t)}$ into the asymptotically derived continuum model (3.30) and noting that $\omega^{2}=k^{2} c_{p}^{2}$ we obtain the following dispersion relation:

$$
c_{p}^{2}=\left(\frac{2 D h}{\Gamma}-\frac{\varepsilon^{2} D h^{3} k^{2}}{3 \Gamma^{2}}\right) /\left(\frac{\varepsilon^{2} k^{2}}{c^{2}}\left[\frac{3 h^{2}+2 D h^{3}}{6 \Gamma^{2}}-\frac{h^{3}}{3 \Gamma}\right]+\rho\right) .
$$

(2) In order to obtain the dispersion relations for the variationally derived continuum model (4.8), (4.9) we first invert the stress-strain constitutive relations (4.9) and combine this with the equations of motion (4.8) to obtain

$$
\begin{aligned}
& {\left[\begin{array}{ccc}
a_{11} \frac{\partial^{2}}{\partial x^{2}} & -\frac{2}{3} a_{12} \frac{\partial}{\partial x} & \frac{4}{45} \varepsilon^{2} h^{2} a_{12} \frac{\partial^{2}}{\partial x^{2}} \\
\frac{2}{3} a_{12} \frac{\partial}{\partial x} & \frac{2}{15} \varepsilon^{2} h^{2} a_{33} \frac{\partial^{2}}{\partial x^{2}}-\frac{4}{9} a_{22} & \left(\frac{4}{15} \varepsilon^{2} h^{2} a_{33}+\frac{8}{135} \varepsilon^{2} h^{2} a_{22}\right) \frac{\partial}{\partial x} \\
\frac{4}{45} \varepsilon^{2} h^{2} a_{12} \frac{\partial^{2}}{\partial x^{2}} & \left(\frac{4}{15} \varepsilon^{2} h^{2} a_{33}+\frac{8}{135} \varepsilon^{2} h^{2} a_{22}\right) \frac{\partial}{\partial x} & \frac{16}{2025} \varepsilon^{4} h^{4} a_{22} \frac{\partial^{2}}{\partial x^{2}}-\frac{24}{45} \varepsilon^{2} h^{2} a_{33}
\end{array}\right]\left\{\begin{array}{l}
u_{0} \\
\psi \\
\phi
\end{array}\right\}} \\
& \quad=\rho\left\{\begin{array}{c}
\frac{\partial^{2} u_{0}}{\partial t^{2}} \\
\frac{1}{3} \varepsilon^{2} h^{2} \frac{\partial^{2} \psi}{\partial t^{2}} \\
\frac{4}{45} \varepsilon^{4} h^{4} \frac{\partial^{2} \phi}{\partial t^{2}}
\end{array}\right\},
\end{aligned}
$$

where the matrix

$$
\left[\begin{array}{ccc}
a_{11} & a_{12} & 0 \\
a_{21} & a_{22} & 0 \\
0 & 0 & a_{33}
\end{array}\right]
$$


is defined as the inverse of (see (4.9))

$$
\left[\begin{array}{ccc}
\frac{1}{E}+\frac{1}{2 D h} & \frac{1}{3 D h} & 0 \\
\frac{1}{3 D h} & \frac{4}{45 E}+\frac{2}{9 D h} & 0 \\
0 & 0 & \frac{4}{7 E}
\end{array}\right]
$$

Substituting $u_{0}=U e^{i(k x-\omega t)}, \psi=\psi_{0} e^{i(k x-\omega t)}$, and $\phi=\phi_{0} e^{i(k x-\omega t)}$ into Eq. (5.2) and setting $\omega^{2}=k^{2} c_{p}^{2}, \psi_{0}=\frac{k \Psi}{i}$, and $\phi_{0}=k^{2} \Phi$ we obtain the eigenvalue problem

$$
\begin{aligned}
& {\left[\begin{array}{ccc}
a_{11} & \frac{2}{3} a_{12} & \frac{4}{45} \varepsilon^{2} h^{2} k^{2} a_{12} \\
\frac{2}{3} a_{12} & \frac{2}{15} \varepsilon^{2} h^{2} k^{2} a_{33}+\frac{4}{9} a_{22} & \left(\frac{4}{15} a_{33}+\frac{8}{135} a_{22}\right) \varepsilon^{2} h^{2} k^{2} \\
\frac{4}{45} \varepsilon^{2} h^{2} k^{2} a_{12} & \left(\frac{4}{15} a_{33}+\frac{8}{135} a_{22}\right) \varepsilon^{2} h^{2} k^{2} & \frac{16}{2025} \varepsilon^{4} h^{4} k^{4} a_{22}+\frac{24}{45} \varepsilon^{2} h^{2} k^{2} a_{33}
\end{array}\right]\left\{\begin{array}{c}
U \\
\Psi \\
\Phi
\end{array}\right\}} \\
& =\rho c_{p}^{2}\left\{\begin{array}{c}
U \\
\frac{1}{3} \varepsilon^{2} h^{2} k^{2} \Psi \\
\frac{4}{45} \varepsilon^{4} h^{4} k^{4} \Phi
\end{array}\right\}
\end{aligned}
$$

Analysis of the preceding equations ${ }^{2}$ leads to the following result:

Proposition 5.1. The dispersion relations obtained from (i) the asymptotically derived model (cf. 5.1), (ii) the variationally derived continuum model (cf. 5.3), and (iii) the exact solution (cf. Appendix) are all identical up to $O\left(\varepsilon^{2}\right)$; i.e., the equations (5.1), (5.3), and (A.3) all lead to the dispersion relation

$$
\frac{c_{p}^{2}}{c_{0}^{2}}=1-\frac{\varepsilon^{2} k^{2}}{3(1+2 D)^{2}}+O\left(\varepsilon^{4} k^{4}\right),
$$

where

$$
c_{0}^{2}=\frac{2 D}{1+2 D} \text {. }
$$

Proof. This result can easily be verified for Eqs. (5.1) and (A.3) by taking Taylor expansions about $\varepsilon k=0$. In order to solve the eigenvalue problem (5.3), we let $\bar{\phi}=\varepsilon \phi$ and write this equation in the form

$$
\left(A_{0}+\varepsilon k A_{1}+\varepsilon^{2} k^{2} A_{2}\right) p=\lambda\left(B_{0}+\varepsilon^{2} k^{2}\right) p
$$

where $\lambda$ is used here for $c_{p}^{2}$. We expand the eigenvalue and eigenfunction as

$$
\begin{aligned}
& \lambda=\lambda_{0}+\varepsilon k \lambda_{1}+\varepsilon^{2} k^{2} \lambda_{2}+\cdots, \\
& p=p_{0}+\varepsilon k p_{1}+\varepsilon^{2} k^{2} p_{2}+\cdots .
\end{aligned}
$$

In the limit as $\varepsilon \rightarrow 0$, Eq. (5.4) leads to the eigenvalue problem for $\lambda_{0}$ :

$$
\left(A_{0}-\lambda_{0} B_{0}\right) p_{0}=0 .
$$

\footnotetext{
${ }^{2}$ For this purpose we use the signal wavelength, the matrix modulus, and the matrix density for scaling various quantities (see Sec. 2) so that $h, E$, and $\rho$ are all unity.
} 
On equating like powers of $\varepsilon k$ we obtain from (5.4) the following sequence of problems involving the same singular operator:

$$
\begin{gathered}
\left(A_{0}-\lambda_{0} B_{0}\right) p_{1}=\left(\lambda_{1} B_{0}-A_{1}\right) p_{0}, \\
\left(A_{0}-\lambda_{0} B_{0}\right) p_{2}=\left(\lambda_{0} B_{2}-\lambda_{2} B_{0}-A_{2}\right) p_{0}+\left(\lambda_{1} B_{0}-A_{1}\right) p_{0} .
\end{gathered}
$$

By noting that $A_{0}-\lambda_{0} B_{0}$ is symmetric, so that its range is orthogonal to $p_{0}$, and requiring that each of the right-hand sides of $(5.5 \mathrm{a}, \mathrm{b})$ be in its range, we obtain the desired result.

Figure 2, which depicts the dispersion relations for the acoustic mode obtained from the various theories for the case wherein the parameter $\frac{E}{D h}=10$, bears out the aforementioned result. The variationally derived model also allows the propagation of waves in the so-called optical modes which are characterized by the unboundedness of the phase velocity in the limit of vanishing wave number. In Fig. 3, we compare the results for the dispersion of lowest optical mode based on this model with the exact solution. Evidently, even for this mode the theory provides a highly accurate model of dispersion.

Moreover, the results indicate that for relatively large wave numbers there is rather good agreement between the dispersion relations predicted by the continuum models and the exact solutions. It thus appears that the models may also be adequate for

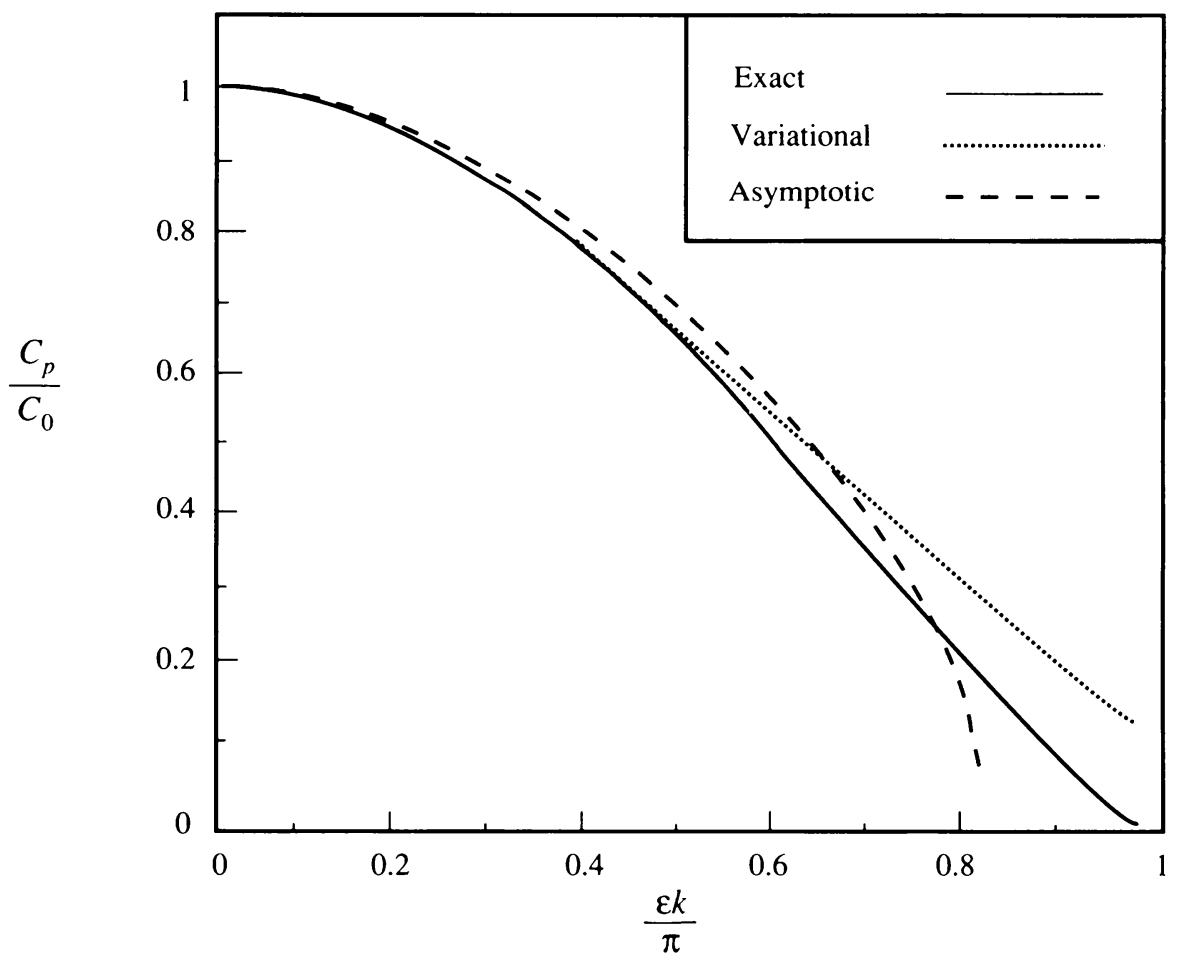

Fig. 2. Dispersion curve for the acoustic mode 


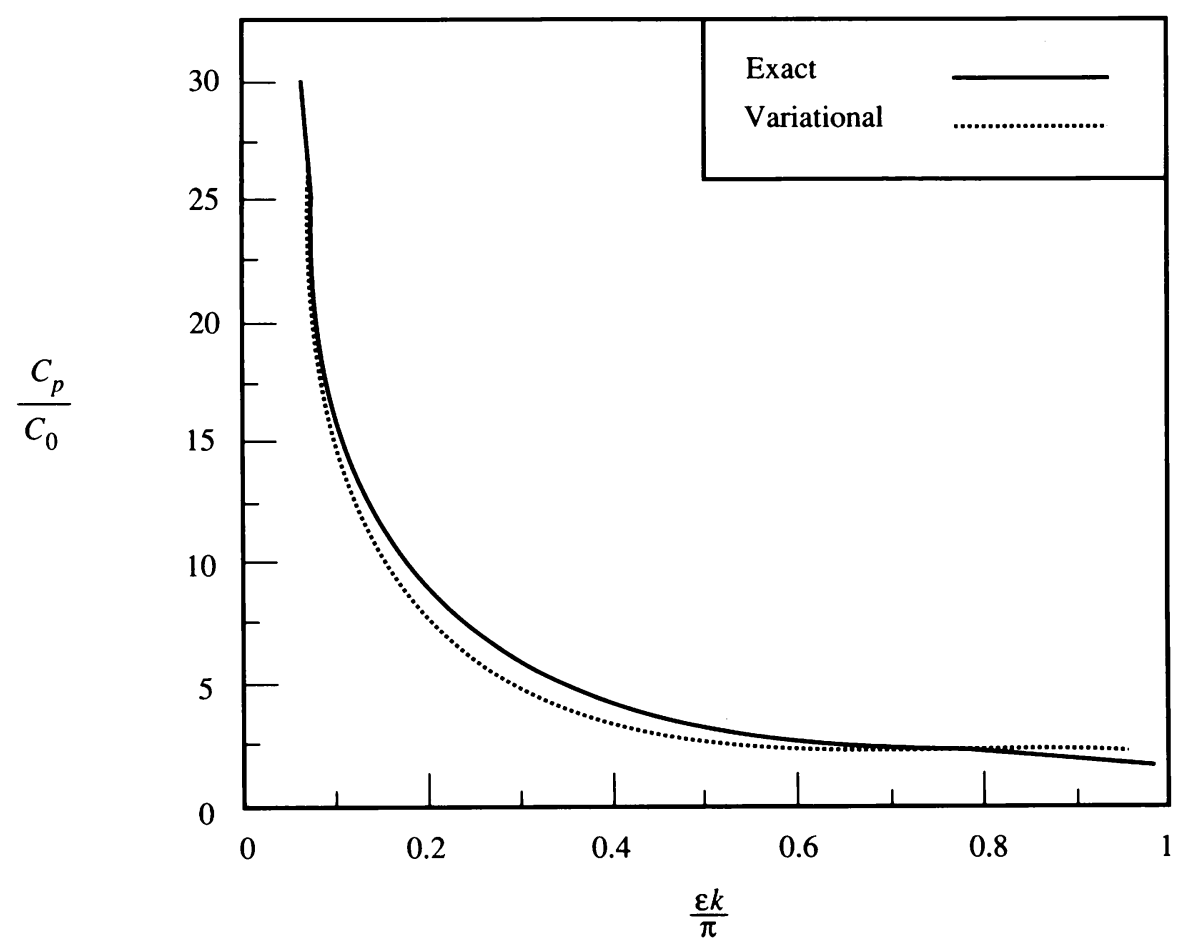

Fig. 3. Dispersion curve for the first optical mode

problems wherein the signal wavelengths are as small as up to two times the cell dimension.

Appendix. The exact dispersion relation for harmonic-wave propagation through the jointed medium is obtained by assuming solutions of the form:

$$
\begin{aligned}
& u(x, t)=U(x) e^{i(k x-\omega t)}, \\
& \sigma(x, t)=\Sigma(x) e^{i(k x-\omega t)}
\end{aligned}
$$

for the scaled version of equations (2.1)-(2.4). Here, $U(x)$ and $\Sigma(x)$ are periodic functions of $x$, as dictated by Floquet Theory.

Substitution of (A.1), (A.2) into (2.1)-(2.4) together with the conditions of periodicity and the existence of nontrvial solutions leads to the dispersion relation

$$
\kappa v \sin (2 \kappa v)=2 \mathscr{E} \sin (\kappa(1+v)) \sin (\kappa(1-v)),
$$

where

$$
\kappa=\varepsilon k h, \quad v=c_{p} \sqrt{\frac{\rho}{E}}, \quad \mathscr{E}=\frac{2 D h}{E} .
$$




\section{REFERENCES}

[1] J. B. Keller, Effective behavior of heterogeneous media, Statist. Mech. and Statist. Meth. in Theory and Appl. (U. Landman, ed.), Plenum Press, New York, 1977, p. 631

[2] I. Babuska, Solution of interface problems by homogenization. I, II, SIAM J. Math. Anal. 7, 603-634, 635-645 (1976)

[3] A. Bensoussan, J. L. Lions, and G. Papanicolaou, Asymptotic Analysis of Periodic Structures, North-Holland, Amsterdam, 1978

[4] J. M. Burgers, On some problems of homogenization, Quart. Appl. Math. XXXV, 421-434 (1978)

[5] E. Sanchez-Palencia, Non-homogeneous media and vibration theory, Lecture Notes in Phys., vol. 127, Springer-Verlag, Berlin, 1980

[6] G. A. Hegemier, et al., On construction of mixture theories for composite materials by the method of multi-variable asymptotic expansion, Cont. Models of Discrete Systems, Univ. of Waterloo Press, 1980, pp. 423-441

[7] A. Maewal, Construction of models of dispersive elastodynamic behavior of periodic composites: $A$ computational approach, Comp. Meth. Appl. Mech. Engrg. 57, 191-205 (1986)

[8] F. Santosa and W. W. Symes, A dispersive effective medium for wave propagation in periodic composites, SIAM J. Appl. Math. 51, 984-1005 (1991)

[9] H. Murakami and G. A. Hegemier, Development of a nonlinear continuum model for wave propagation in jointed media: Theory for single joint set, Mech. Mat. 8, 199-218 (1989)

[10] J. P. Keener, Principles of Applied Mathematics, Addison-Wesley, New York, 1988

[11] W. Rudin, Real and Complex Analysis, 3rd. ed., McGraw-Hill, New York, 1987

[12] J. P. Aubin, Approximation of Elliptic Boundary-Value Problems, Wiley-Interscience, New York, 1972 